\title{
Tumor sincrónico de endometrio y ovario. Patología infrecuente en ginecología oncológica
}

\author{
Drs. Alba Rodríguez Pérez, ${ }^{1}$ Jara Gallardo Martínez, ${ }^{1}$ Zoraida Frías Sánchez, ${ }^{2}$ \\ Manuel Pantoja Garrido, ${ }^{1}$ María del Valle Aguilar Martín, ${ }^{1}$ Inmaculada Rodríguez Jiménez, ${ }^{1}$ \\ Mario Roquette Mateos, ${ }^{3}$ Álvaro Gutiérrez Domingo ${ }^{4}$
}

\begin{abstract}
RESUMEN
El cáncer de endometrio se mantiene como el tumor más frecuente del tracto ginecológico en las mujeres de Estados Unidos de Norteamérica (EE.UU). El hallazgo simultáneo de un cáncer de endometrio y ovario, como tumor sincrónico, alcanza el $5 \%$ - $10 \%$ del total de estas neoplasias, tratándose, por tanto, de una rara entidad. La consideración de metástasis restringidas o pseudometástasis para definir la diseminación de este tipo de tumoraciones, toma mayor relevancia hoy en día gracias a los últimos avances en el campo de la inmunohistoquímica y biología molecular. En este artículo se expone el caso de una paciente de 57 años diagnosticada inicialmente de un carcinoma de endometrio estadio IIIA de la Federación Internacional de Ginecología y Obstetricia (FIGO); siendo posteriormente un tumor sincrónico de endometrio estadio IB y de ovario IC, pese a la sospecha prequirúrgica de tumor metastásico.
\end{abstract}

Palabras clave: Cáncer de endometrio, Cáncer de ovario, Tumores sincrónicos, Neoplasia primaria múltiple, Metástasis.

\section{SUMMARY}

Endometrial cancer remains the most common gynecological tumor in women in the United States of America. The simultaneous finding of endometrial and ovarian neoplasm, as a synchronous tumor, accounts for about $5-10 \%$ of endometrial and ovarian tumors. Therefore, is a rare entity. Consideration as restricted metastases or pseudometastasis to define the spread of this kind of tumor, is becoming more relevant nowadays thanks to the latest advances in the field of immunohistochemistry and molecular biology. In this article we present the case of a 57-year-old patient initially diagnosed with FIGO stage IIIA endometrial carcinoma; subsequently, it was a synchronous endometrial tumor stage IB and IC ovary, despite the presurgical suspicion of a metastatic tumor.

Keywords: Endometrial Neoplasm, Ovarian Neoplasm, Synchronous Neoplasm, Multiple Primary Neoplasm, Neoplasm Metastasis.

\section{INTRODUCCIÓN}

El tumor sincrónico se define como el desarrollo

\footnotetext{
${ }^{1}$ Unidad de Gestión Clínica (UGC) de Obstetricia y Ginecología del Hospital Universitario Virgen (HUV) Macarena de Sevilla (España). ${ }^{2}$ UGC de Ginecología y Patología Mamaria del HUV del Rocío de Sevilla (España) ${ }^{3}$ UGC de Radiología del HUV Macarena de Sevilla (España). ${ }^{4}$ UGC de Anatomía Patológica del HUV Macarena de Sevilla (España).
}

primario de dos o más tumoraciones en una misma paciente, muy cercanas en el tiempo. La definición aún no ha sido consensuada, siendo objeto de controversia hoy en día. Estas neoplasias se caracterizan por un pronóstico más favorable, ya que la naturaleza metastásica no puede ser demostrada (1). En el campo de la ginecología, los tumores sincrónicos son una rara entidad observada en el $1 \%$ - $2 \%$ de las mujeres que 
desarrollan un cáncer ginecológico siendo los más frecuentes los que implican al cáncer de endometrio y ovario (SEOC), en torno al $50 \%$ - $70 \%$ (2). En un $5 \%$ de las pacientes con cáncer de endometrio y en un $10 \%$ de las que desarrollan una neoplasia de ovario, se objetiva la existencia de un tumor sincrónico (2-4). Afecta generalmente a mujeres jóvenes, premenopáusicas, nulíparas y, en un gran porcentaje de los casos, obesas $(3,5)$. Estas características se contraponen al desarrollo independiente, que generalmente afecta a mujeres posmenopáusicas entre la sexta y séptima década de la vida. Las manifestaciones clínicas son similares a las de los tumores independientes; generalmente se expresan inicialmente a través de la lesión endometrial, en forma de sangrado anómalo, siendo la sintomatología secundaria a la neoplasia ovárica mayoritariamente inespecífica (3). El diagnóstico de esta patología es controvertido. La clasificación histológica clásica según los criterios de Ulbright y Roth (6), modificada por Scully (7) en 1998, a menudo supone un desafío, ya que suelen ser lesiones con el mismo subtipo histopatológico lo que dificulta aún más su diferenciación respecto a las lesiones metastásicas. Los avances en el campo de la inmunohistoquímica y biología molecular permiten una mejor aproximación al diagnóstico que apoya el abandono del concepto de tumores primarios independientes, hacia la concepción de neoplasias clonalmente relacionadas con extensión metastásica (8-12).

Llegados a este punto, es importante reportar casos que permitan obtener una visión más clara de esta infrecuente patología y así poder diagnosticarlas precozmente, mejorando, por tanto, su manejo terapéutico, pronóstico y permitiendo, además, la preservación de la fertilidad en las pacientes con deseo genésico. A continuación, se expone el caso de una mujer diagnosticada de dos tumores ginecológicos malignos de endometrio y ovario de alto grado, catalogados como sincrónicos e independientes.

\section{CASO CLÍNICO}

Se presenta el caso de una paciente de 57 años que acudió a las consultas externas del Hospital Universitario Virgen Macarena de Sevilla (España), por sangrado posmenopáusico. Entre sus antecedentes médicos destacaban la hipertensión arterial $\mathrm{y}$ bronquitis crónica, ser fumadora y haber sido intervenida de una faquectomía. En cuanto a los antecedentes ginecoobstétricos, había presentado la menarquia a los 14 años, dos gestaciones finalizadas mediante parto vaginal y la menopausia a los 47 años. La paciente refería sangrado genital de 6 meses de evolución, sin episodios de sangrado previos. La exploración genital objetivó un rectocele grado II y un cérvix bien epitelizado, desplazado hacia arriba por un útero notablemente aumentado de tamaño, consistente y fijo. En la ecografía transvaginal, se visualizaba un notable e irregular engrosamiento endometrial de $47 \mathrm{~mm}$, con miometrio adelgazado, sin ser posible la visualización de los anexos. Ante tales hallazgos, se realizó una biopsia endometrial y se derivó a la consulta de ginecología oncológica. El informe anatomopatológico mostraba un resultado compatible con adenocarcinoma endometrioide con patrón villoglandular, por lo que se solicitó una resonancia magnética nuclear (RMN) que se informó como: leiomiomas de localización intramural y submucosa. Engrosamiento e irregularidad endometrial con ensanchamiento del canal endometrial y patrón de realce tras a administración de contraste, compatible con carcinoma endometrial con afectación secundaria de ambos anexos, observándose un diámetro transverso máximo de la lesión referida de 11 $\mathrm{cm}$. Ante tales hallazgos, el equipo de ginecología oncológica indicó la realización de una cirugía para estadificar, que consistió en una exploración minuciosa de la cavidad abdominopélvica con lavado peritoneal e histerectomía total con doble anexectomía, omentectomía, apendicectomía, linfadenectomía 
pélvica y aortocava, vía laparotomía. La intervención se realizó sin complicaciones destacando como hallazgos intraoperatorios la existencia de 3,5 litros de líquido ascítico de aspecto seromucinoso y una masa abigarrada de contenido mucinoso y sólido de $12 \times 12 \mathrm{~cm}$ en ovario derecho, que se extendía hacia la cara posterior uterina. La evolución posoperatoria fue favorable, recibiendo la paciente el alta hospitalaria a los 3 días de la intervención. El análisis histológico de la pieza quirúrgica evidenció la presencia de un adenocarcinoma endometrioide de endometrio con diferenciación villoglandular y mucinosa apical, con invasión profunda miometrial (mayor del $50 \%$ ) $\mathrm{y}$ del istmo uterino, pero sin afectación parametrial; mientras que el estudio del anexo derecho reportó adenocarcinoma endometrioide con diferenciación mucinosa, que invadía y producía disrupción de la cápsula. El estudio inmunohistoquímico reveló que las células neoplásicas expresaban en ambas neoplasias citoqueratinas AE1/AE3, CK7 (focal), AME, CEA (apicalmente y en las luces glandulares) y estrógenos focalmente. La citoqueratina CK20, p53 y PETEN fueron negativos. El resto de las muestras quirúrgicas se encontraron libres de neoplasia. No se observaron alteraciones histológicas en las trompas de Falopio, ovario izquierdo, apéndice, epiplón y ganglios aortocavos e iliacos (Figura 1). Así pues, se confirmó el diagnóstico de dos tumores independientes y sincrónicos, un estadio IB de endometrio y IC de ovario, según la clasificación de la Federación
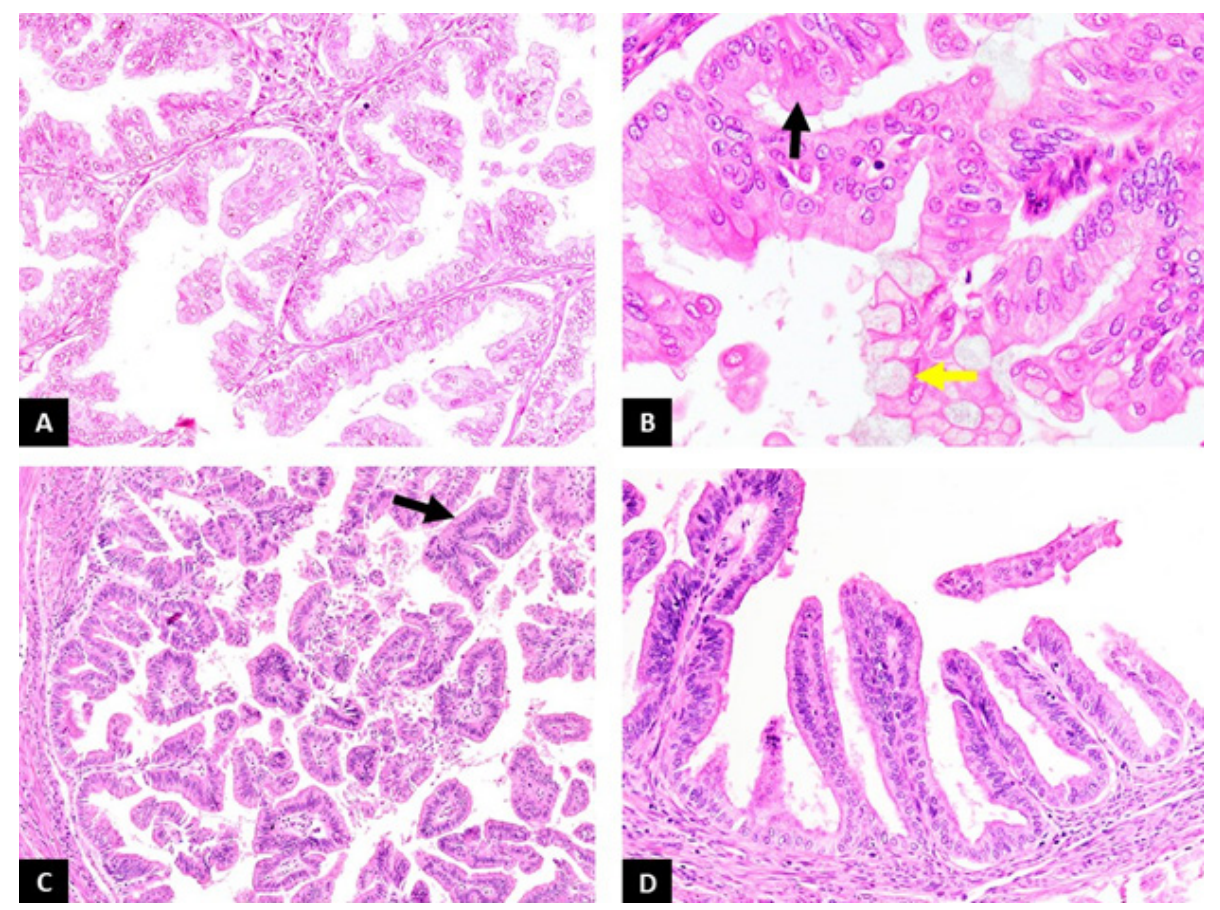

Figura 1: Adenocarcinoma sincrónico de ovario con diferenciación mucinosa (endometrioide) y adenocarcinoma endometrioide de uterino con diferenciación villoglandular.

A) Adenocarcinoma endometrioide ovárico: tumor compuesto por glándulas sin estroma interpuesto (back to back) que asemeja al carcinoma endometrioide de endometrio (HE,10x). B) A mayor aumento, mayor detalle de las características citológicas de la neoplasia: células columnares con citoplasma eosinófilo (flecha negra) y células mucinosas (flecha amarilla) (HE,10x). C) Adenocarcinoma endometrioide del útero: tumor con una clara arquitectura vellosa. Obsérvense las proyecciones digitiformes de las que se compone la neoplasia (flecha) (HE,10x). D) A mayor aumento, detalle de las vellosidades: se trata de papilas finas sin proliferación epitelial exagerada y que conservan la polaridad nuclear (HE,20x). 

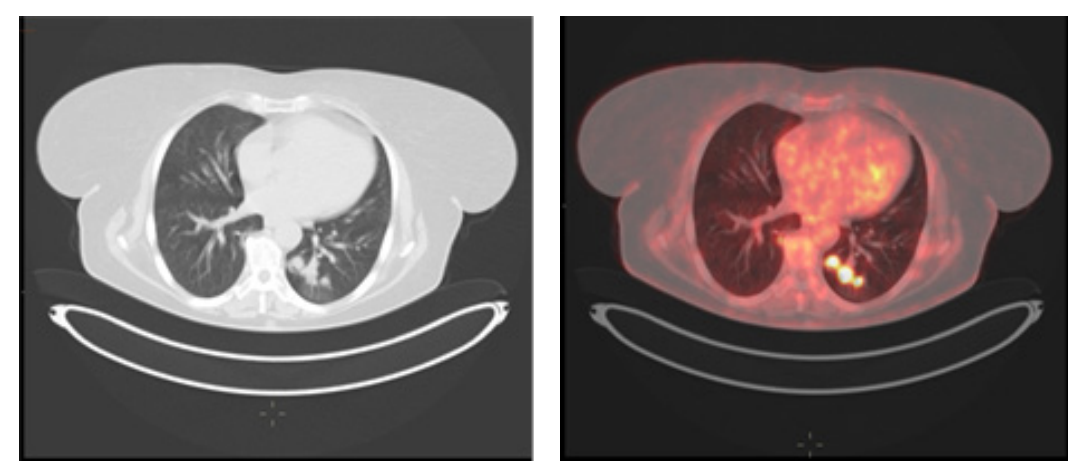

Figura 2: Tomografía computarizada (TC) y tomografía por emisión de positrones (PET-TC) corte axial de tórax: en la imagen de la izquierda se observan los nódulos pulmonares localizados en el lóbulo inferior izquierdo; a la derecha se observan los nódulos con depósitos hipermetabólicos de moderada intensidad.

Internacional de Obstetricia y Ginecología (FIGO). El comité interdisciplinar de tumores ginecológicos decidió tratamiento adyuvante con quimioterapia, siguiendo un plan terapéutico de 4 ciclos de paclitaxel/ carboplatino, y posterior radioterapia pélvica externa y braquiterapia vaginal. Tras el mismo, la paciente presentó una respuesta positiva al tratamiento con un seguimiento posterior normal. Llevaba 8 años en periodo libre de enfermedad, con revisiones multidisciplinares, sin sospechas ni evidencias de recidivas. Sin embargo, en un estudio realizado en marzo de 2020 para la evaluación de un cuadro de anemia presentado por la paciente, se observó la presencia de nódulos pulmonares sugestivos de malignidad en una tomografía axial computerizada (TAC) y tomografía por emisión de positrones (PETTC) (Figura 2). Dado el antecedente de neoplasia ginecológica, se realizó un estudio para descartar su origen metastásico. Tras la exéresis quirúrgica de los nódulos pulmonares se confirmó la presencia de un carcinoma escamoso de pulmón, excluyendo la naturaleza metastásica de las lesiones.

\section{DISCUSIÓN}

Los tumores sincrónicos ginecológicos son una rara entidad. La incidencia descrita en las últimas series abarca del $2 \%$ al 8,5 \% de las neoplasias ginecológicas (13). El cáncer de endometrio es el más prevalente de los tumores del tracto genital femenino, mientras que el de ovario es el más letal. La mayoría de los estudios describen medias de edad entre 40 y 50 años (14), encontrándose la paciente descrita fuera de este rango de edad. En un $55 \%$ de los casos son mujeres posmenopáusicas, al igual que esta paciente. Ante el hallazgo simultáneo de dos lesiones en el tracto genital femenino, el clínico debe plantearse diferentes posibilidades: que sean lesiones metastásicas e identificar el tumor primario, que sean lesiones sincrónicas independientes o que formen parte de un síndrome hereditario como el Síndrome de Lynch (4, 12).

Hoy en día se emplean los criterios histopatológicos de Scully y cols. (7) para describir los aspectos que permiten diferenciar un tumor sincrónico de una metástasis, siendo utilizados por la mayoría de servicios de anatomía patológica, aunque aún no han sido validados internacionalmente. En un $90 \%$ de los casos se trata de lesiones de bajo grado compatibles con adenocarcinoma endometrioide (70 \% de los mismos) $(1,4,12)$, hallazgos encontrados en el caso clínico expuesto. Las diferencias histológicas para el cáncer de endometrio son: ausencia de invasión 
o afectación superficial del miometrio, ausencia de invasión vascular linfática, ausencia de extensión parametrial o presencia de hiperplasia atípica. Para el cáncer de ovario, deben cumplirse las siguientes premisas: unilateralidad, afectación contralateral de la superficie ovárica, localización hiliar, ausencia de extensión capsular o extracapsular, y presencia de endometriosis (7). Sin embargo, en la práctica no siempre es fácil distinguir las lesiones sincrónicas de las metastásicas, sobre todo en aquellos casos que comparten características independientes y metastásicas (como pueden ser tumor endometrial de grandes dimensiones, bilateralidad, ausencia de endometriosis, etc.) (7).

En cuanto a la expresión de proteínas, los tumores sincrónicos (en comparación con el carcinoma de ovario endometrioide aislado), presentan déficit de MLH1, PMS2, PTEN, aunque esto no se traduce en diferencias pronósticas (15). En el caso presentado, se puede confirmar el déficit de proteína PTEN como identificador de tumor sincrónico.

El estudio de Turashvili y cols. (1) sugiere que se puede aplicar una serie de sencillos criterios clínicos e histológicos para estratificar el riesgo de estas pacientes, permitiendo una clasificación, de acuerdo con su comportamiento, que consiga una aproximación diagnóstica y terapéutica más sencilla. Definen como bajo riesgo aquellas neoplasias de histología endometrioide (independientemente de la invasión miometrial o de la afectación linfática), así como carcinomas no endometrioides sin invasión vascular, con una afectación miometrial $<50 \%$. Por el contrario, serían de alto riesgo los carcinomas no endometrioides con invasión miometrial superior al $50 \%$, con o sin invasión nodular. Según se ha observado, los criterios diagnósticos implican el estudio de la pieza quirúrgica, sin adelantarse a la propia cirugía para su determinación. El grupo de Moro y cols. (13), a partir de 131 casos analizados
(51 neoplasias sincrónicas y 80 metastásicas), propone una serie de diferencias sonomorfológicas que pueden orientar el diagnóstico prequirúrgico, como que los tumores sincrónicos con mayor frecuencia presentan tumoraciones ováricas unilaterales de ecogenicidad multilocular-sólida o sólida asociada a una lesión endometrial, que suele ser pequeña, hiperecogénica, sin invasión miometrial y con escasa vascularización. Por el contrario, las lesiones metastásicas ováricas suelen ser bilaterales y sólidas, mientras que la lesión endometrial presenta un gran tamaño, con invasión miometrial y abundante vascularización con patrón multivaso. Las últimas clasificaciones consideran que tumores de endometrio de bajo grado endometrioides con una neoplasia secundaria unilateral en el ovario, deben definirse como un estadio "IIIA- simulating independent primary tumors", recomendando un manejo conservador (16).

La biología de estos tumores sigue siendo controvertida, parece claro que, dado que comparten características morfológicas y moleculares, deben presentar un origen común. En el pasado, autores como Song y cols. (5) apostaban por un ambiente favorecedor de la tumorogénesis, como podría ser el efecto hormonal estrogénico o la endometriosis, dada su mayor incidencia en mujeres premenopáusicas, aunque esta teoría aún no ha sido demostrada. Por otro lado, Eifel y cols. (17) sugieren la existencia de una respuesta conjunta del sistema mülleriano que podría explicar su desarrollo simultáneo. Estudios recientes en los que se secuencian variantes de nucleótidos, así como el exoma de tumores de bajo grado, encuentran similitudes en cuanto a las mutaciones somáticas y el número de copias $(8,11)$. De estos hallazgos se deduce que los carcinomas de bajo grado de tipo endometrioide, al presentar las mismas alteraciones genómicas, comparten un origen clonal y, de ese modo, deben considerarse metástasis dado su origen común. Sin embargo, estos estudios no han podido demostrar cuál es la dirección de la metástasis, es decir, cuál es el 
tumor primario del que parten las células metastásicas; seguramente surjan desde el endometrio para alcanzar finalmente el ovario, basándose en la capacidad del ovario como receptor de metástasis de numerosos cánceres (gástrico, mama) (9).

Las neoplasias endometriales con afectación del ovario pueden dividirse en dos grupos, el primero con un comportamiento asintomático, como si fueran dos primarios (seguramente producto de metástasis transtubáricas) $\mathrm{y}$, en segundo lugar, como carcinomas de alto riesgo, con extensión por las vías tradicionales (transcelómica), que requieren de la adquisición de numerosas capacidades prometastásicas (9). El fenómeno de metástasis restringidas, o pseudometástasis, se emplea para definir la diseminación de los tumores sincrónicos de endometrio y ovario, de buen pronóstico. Esta teoría explica la diseminación de las células tumorales que se desprenden de la lesión primaria, probablemente vía transtubárica, alcanzando las áreas anexiales sin poder sobrepasarlas, ya que requieren de la presencia de un microambiente determinado. Las células deben tener la capacidad de desprenderse del tumor primario sin sufrir apoptosis (anoikis), propagarse y colonizar el microambiente ovárico, sin sobrepasarlo $(9,11)$. Este fenómeno se observa también en las neoplasias asociadas al virus del papiloma humano (VPH) (cérvix, vulva) y en algunas neoplasias uroteliales (8). Ante esta concepción, cabría pensar que las mujeres con tumores sincrónicos presentan mayor susceptibilidad para el desarrollo de neoplasias, asociadas o no a un síndrome tumoral genético como el Síndrome de Lynch, sin embargo, la probabilidad se estima en torno a un $7 \%$, no muy diferente de la de las pacientes con un carcinoma endometrial único (4).

En cuanto a la clínica de los tumores sincrónicos, generalmente predominan las manifestaciones de uno de ellos, diagnosticándose posteriormente de forma accidental, la neoplasia sincrónica. En el caso expuesto, la sintomatología que presentaba la paciente fue de carcinoma endometrial (sangrado posmenopáusico), que se confirmó en el estudio mediante biopsia prequirúrgica; mientras que el hallazgo de la tumoración en el ovario partió del estudio de extensión con prueba de imagen, confirmándose durante la cirugía y en el posterior estudio histológico.

Respecto al tratamiento, la concepción de metástasis restringidas afecta a la consideración de resecabilidad del tumor, así como al pronóstico, intentando no sobretratar a estas pacientes. El tratamiento quirúrgico sigue siendo la primera opción terapéutica para estas pacientes, mientras que el uso de terapias adyuvantes (quimioterapia y/o radioterapia) es más controvertido. Los estudios publicados no demuestran mejores tasas de supervivencia del tratamiento quirúrgico exclusivo respecto a la adyuvancia, si bien es cierto que suelen ser pacientes que reúnen peores factores pronósticos. Diferentes autores proponen que la adyuvancia debería reservarse para las pacientes que presenten edad avanzada, posmenopáusicas, con tumoraciones de alto grado histológico o enfermedad residual (14); deberían emplearse ciclos de carboplatino/paclitaxel y si la neoplasia endometrial presenta un estadio avanzado, con afectación miometrial y/o parametrial, añadir radioterapia (3). La paciente reunía factores de mal pronóstico por lo que, tras valoración por un comité multidisciplinario, se decidió como adyuvancia a la cirugía un tratamiento con pauta de quimioterapia con carboplatino/paclitaxel y posterior radioterapia pélvica externa y braquiterapia vaginal. Varios estudios muestran que las mujeres con un tumor sincrónico presentan mejor pronóstico que con enfermedad metastásica.

La investigación prospectiva del equipo de Zaino y cols. (18) evidencia unas tasas de supervivencia para tumores sincrónicos del 85,9 \% a los 5 años y del $80,3 \%$ a los 10 años; mientras que para la enfermedad metastásica la supervivencia es del $48 \%$ y $15 \%$, respectivamente. Se han descrito factores pronósticos, como la edad (mejor en jóvenes), la histología 
(tumores endometrioides presentan mejor pronóstico frente a los no endometrioides, sobre todo el subtipo de células claras) (4), estadio tumoral ovárico o la presencia de enfermedad residual; mientras que el papel del estadio endometrial presenta resultados contradictorios (2). Recientemente, niveles elevados de $\mathrm{Ca} 125$ pretratamiento se han definido como un factor pronóstico independiente para la recurrencia y supervivencia de esta patología (5).

Los autores concluyen que el diagnóstico de dos tumoraciones en una paciente debe ser estudiado con cautela para determinar su origen, ya que afectará directamente al pronóstico y tratamiento. Se debe tener en cuenta esta posibilidad diagnóstica, sobre todo en pacientes jóvenes, aunque puede presentarse más tardíamente como en el caso presentado. Parece cada vez más aceptada la consideración de pseudometástasis o metástasis restringidas, sin embargo, es necesario reportar los casos de esta infrecuente patología, permitiéndonos así un mayor conocimiento de esta enfermedad, logrando un mejor manejo de esta.

Los autores declaran no tener ningún conflicto de intereses

\section{REFERENCIAS}

1. Turashvili G, Gómez-Hidalgo NR, Flynn J, Gonen M, Leitao MM, Soslow RA, et al. Risk-based Stratification of Carcinomas Concurrently Involving the Endometrium and Ovary. Gynecol Oncol. 2019; 152 (1): 38 - 45. doi: 10.1016/j.ygyno.2018.10.033.

2. Sozen H, Vatansever D, Iyibozkurt AC, Topuz S, Ozsurmeli M, Salihoglu Y, et al. Clinicopathologic and survival analyses of synchronous primary endometrial and epithelial ovarian cancers. J Obstet Gynaecol Res. 2015; 41 (11): 1813 - 9. doi: 10.1111/jog. 12826.

3. Jain V, Sekhon R, Pasricha S, Giri S, Modi KB, Shrestha E, et al. Clinicopathological characteristics and prognostic factors of synchronous endometrial and ovarian cancersva single-institute review of 43 cases. Int J Gynecol Cancer. 2017; 27 (5): 938 - 46. doi: 10.1097/IGC.0000000000000971.
4. Soliman PT, Broaddus RR, Schmeler KM, Daniels MS, Gonzalez D, Slomovitz BM, et al. Women with synchronous primary cancers of the endometrium and ovary: Do they have Lynch syndrome? J Clin Oncol. 2005; 23 (36): 9344 - 50. doi: 10.1200/ JCO.2005.03.5915.

5. Song T, Seong SJ, Bae DS, Kim JH, Suh DH, Lee $\mathrm{KH}$, et al. Prognostic factors in women with synchronous endometrial and ovarian cancers. Int $\mathrm{J}$ Gynecol Cancer. 2014; 24 (3): 520 - 7. doi: 10.1097/ IGC.0000000000000073.

6. Ulbright TM, Roth LM. Metastatic and independent cancers of the endometrium and ovary: A clinicopathologic study of 34 cases. Hum Pathol. 1985; 16 (1): 28 - 34. doi:10.1016/S0046-8177(85)80210-0.

7. Scully R, Young R, Clement P. Atlas of Tumor Pathology: Tumors of the Ovary, Maldeveloped Gonads, Fallopian Tube, and Broad Ligament. Silver Spring: Amer Registry of Pathology; 1999.

8. Anglesio MS, Wang YK, Maassen M, Horlings HM, Bashashati A, Senz J, et al. Synchronous Endometrial and Ovarian Carcinomas: Evidence of Clonality. J Natl Cancer Inst. 2016; 108 (6): 1 - 5. doi:10.1093/jnci/ djv428.

9. Casey L, Singh N. Metastases to the ovary arising from endometrial, cervical and fallopian tube cancer: recent advances. Histopathology. 2020; 76 (1): 37 - 51. doi: 10.1111/his.13985.

10. Niskakoski A, Pasanen A, Porkka N, Eldfors S, Lassus $\mathrm{H}$, Renkonen-Sinisalo L, et al. Converging endometrial and ovarian tumorigenesis in Lynch syndrome: Shared origin of synchronous carcinomas. Gynecol Oncol. 2018; 150 (1): 92 -8. doi:10.1016/j.ygyno.2018.04.566.

11. Gilks CB, Kommoss F. Synchronous tumours of the female reproductive tract. Pathology. 2018; 50 (2): 214 -21. doi: 10.1016/j.pathol.2017.10.007.

12. Frías-Sánchez Z, Pantoja-Garrido M, Rojo-Novo S, Jiménes Gallardo J, Márquez-Maraver F, Polo-Velasco $\mathrm{A}$, et al. Adenocarcinoma sincrónico de alto grado de endometrio y ovario; a propósito de un caso clínico. Rev Chil Obstet Ginecol. 2018; 83 (4): 394 - 401. doi: $10.4067 / \mathrm{s} 0717-75262018000400394$.

13. Moro F, Leombroni M, Pasciuto T, Trivellizzi IN, Mascilini F, Ciccarone F, et al. Synchronous primary cancers of endometrium and ovary vs endometrial cancer with ovarian metastasis: an observational study. Ultrasound Obstet Gynecol. 2019; 53 (6): 827 - 35. doi: 10.1002/uog.20213.

14. Bese T, Sal V, Kahramanoglu I, Tokgozoglu N, 


\section{TUMOR SINCRÓNICO DE ENDOMETRIO Y OVARIO. PATOLOGÍA INFRECUENTE EN GINECOLOGÍA ONCOLÓGICA}

Demirkiran F, Turan H, et al. Synchronous Primary Cancers of the Endometrium and Ovary with the Same Histopathologic Type Versus Endometrial Cancer with Ovarian Metastasis: A Single Institution Review of 72 Cases. Int J Gynecol Cancer. 2016; 26 (2): 394 - 406. doi:10.1097/IGC.0000000000000600.

15. Kelemen LE, Rambau PF, Koziak JM, Steed H, Köbel M. Synchronous endometrial and ovarian carcinomas: predictors of risk and associations with survival and tumor expression profiles. Cancer Causes Control. 2017; 28 (5): 447 - 57. doi:10.1007/s10552-017-08555.

16. Sociedad Española de Ginecología y Obstetricia. Guía de Asistencia Práctica. Oncoguía SEGO: Cáncer de Endometrio 2016. Prog Obstet Ginecol. 2017; 60 (3): $274-302$.
17. Eifel P, Hendrickson M, Ross J, Ballon S, Martinez A, Kempson R. Simultaneous presentation of carcinoma involving the ovary and the uterine corpus. Cancer. 1982; 50 (1): 163 - 70. doi: 10.1002/1097-0142(19820701)50:1<163::aidcncr2820500131>3.0.co;2-k

18. Zaino R, Whitney C, Brady MF, DeGeest K, Burger RA, Buller RE. Simultaneously detected endometrial and ovarian carcinomas - A prospective clinicopathologic study of 74 cases: A Gynecologic Oncology Group study. Gynecol Oncol. 2001; 83 (2): 355 - 62. doi:10.1006/gyno.2001.6400.

Recibido 15 de junio de 2020 Aprobado 18 de agosto de 2020 